SYPHILIS

\title{
Treatment for syphilis in antenatal care: compliance with the three dose standard treatment regimen
}

\author{
S Mullick, M Beksinksa, S Msomi
}

See end of article for

Sex Transm Infect 2005;81:220-222. doi: 10.1136/sti.2004.011999

authors' affiliations

Correspondence to:

S Mullick, Population

Council, Frontiers in

Reproductive Health, Hyde

Park Lane Manor, EGO01

Edinburgh Gate, Hyde

Park, Box 411744 ,

Craighall 2024,

Johannesburg, South

Africa; smullick@pcjoburg.

org.za

Accepted for publication

3 September 2004

ses of benzathine penicillin $2.4 \mathrm{MU}$ at weekly intervals are

Background: In South Africa, three doses of benzathine penicillin $2.4 \mathrm{MU}$ at weekly intervals are
recommended for treating syphilis in pregnancy. Limited information is available on compliance with the recommended regimen, in terms of time to starting treatment, number of doses, and timing of treatment. Methods: The study was conducted to establish the degree of compliance with treatment for syphilis. Timing of treatment and the titres of the rapid plasma reagin (RPR) positive women were recorded. A retrospective record review was conducted of 18128 antenatal records. These were records of women attending antenatal care clinics in a tertiary hospital catchment area in KwaZulu Natal between February 2001 and January 2002.

Results: Treatment patterns showed that $15.9 \%$ received no treatment, $13.2 \%$ one dose, $5.8 \%$ received two doses, and $64.8 \%$ received three doses. In total, 188 women (1.03\%) were found to be RPR positive. Of these $36 \%$ were found to be high titre positives (titre $\geqslant 1: 8$ ).

Conclusion: Completed treatment was significantly associated with age of gestation at first visit $(p=0.029)$, with women attending later in pregnancy less likely to receive all three doses of treatment.

$\mathrm{S}$ yphilis is known to pose a risk to pregnancy. If left untreated syphilis can lead to negative outcomes including spontaneous abortion, stillbirth, and perinatal death. Syphilis has also been found to facilitate the risk of HIV transmission. ${ }^{1}$ Syphilis still remains a problem in both the developed as well as developing world. In Africa the antenatal syphilis prevalence has been found to be in the range of $3 \%$ to $17 \% .^{2-5} \mathrm{~A}$ need for improvement in syphilis screening during antenatal care (ANC) has been identified by some of these studies. ${ }^{3}$ Prenatal screening and treatment for positive cases have also been improved. In addition, screening for syphilis in pregnancy has been found to be a cost effective intervention in a number of different settings. ${ }^{67}$

South Africa has one of the fastest growing HIV and sexually transmitted infection (STI) epidemics in the world. Data from the 2002 national antenatal survey showed that on average $26.5 \%$ of women attending ANC clinics were infected with HIV and 3.2\% with syphilis. ${ }^{5}$ Congenital syphilis is a major cause of perinatal mortality in South Africa and in one study $10 \%$ of stillbirths were found to be the result of congenital syphilis. ${ }^{8}$ Among deliveries of women with untreated syphilis, around one third will result in stillbirth or infant death and another third in congenital syphilis, while only one third of infants will be uninfected. ${ }^{9}$

Injections of benzathine penicillin are the accepted treatment for syphilis in pregnancy, but treatment regimens vary. Several countries in Africa have moved to a one dose policy ${ }^{2} 6$ while others like South Africa recommend a three dose regimen. ${ }^{10}$ In practice, however, testing is usually not available on site, necessitating four visits if a woman is seropositive, in order to be screened, collect her results, and complete treatment.

In one South African study conducted in 1995 only $49 \%$ of women screened received all three doses of penicillin. ${ }^{11}$ Three years later there was little improvement, with $61 \%$ of women receiving all three doses, $13 \%$ receiving one dose, and 19\% not receiving any treatment at all. ${ }^{12}$ An earlier study in a South African teaching hospital found that only $22 \%$ of women received a full course of treatment. ${ }^{4}$
Compliance with treatment for syphilis is also not routinely monitored as part of the antenatal programme in South Africa. One study highlighted the challenges with other aspects of the syphilis screening programme in antenatal care. This study showed that despite a policy to screen all women for syphilis in pregnancy there are challenges to the implementation of this aspect of antenatal care. ${ }^{13}$

There is no recent information on compliance with the three dose regimen, particularly in an urban/periurban setting. There has been limited investigation of the main factors associated with compliance.

\section{METHODS}

This study was conducted in Prince Mshiyeni Memorial Hospital (PMMH), a large urban referral and delivery centre in the township of Umlazi, south of Durban, in KwaZulu Natal. This hospital is a referral centre for 21 clinics at Umlazi and surrounding locations and handles an average of 18000 deliveries in a year.

The clinics that feed to PMMH do not have onsite rapid plasma reagin (RPR) testing equipment. Blood samples are drawn from women attending antenatal care for the first time during their pregnancy. These blood specimens are sent to PMMH laboratory for testing. The results are recorded in the hospital's register and copies of the results sent to clinics for treatment of positive clients.

A retrospective 1 year record review was conducted of all available antenatal records filed at the hospital between February 2001 and January 2002. All records were from women receiving antenatal care from the clinics feeding to PMMH or from the hospital itself. Not all records retained were from women who delivered at the hospital. If the woman was admitted during her pregnancy for conditions such as pregnancy induced hypertension, lower abdominal pain, antepartum haemorrhage, anaemia, threatened

Abbreviations: ANC, antenatal care; PMTCT, prevention of mother to child transmission; RPR, rapid plasma reagin; STI, sexually transmitted infections 
Table 1 Total number of injections of benzathine penicillin received by women diagnosed as RPR positive

\begin{tabular}{lrc}
\hline Number of injections & \multicolumn{1}{l}{$\%$} & $\mathbf{n}=186^{*}$ \\
\hline None & 15.9 & 30 \\
One & 13.2 & 23 \\
Two & 5.8 & 11 \\
Three & 64.8 & 122 \\
\hline
\end{tabular}

${ }^{*}$ Two records missing for number of injections.

abortion, and other reasons her record was retained at the hospital.

Data from antenatal cards were extracted by two trained nurses using a structured data collection sheet. This collected information on antenatal clinic attended, gestational age at first visit, RPR result and titre (if available), treatment given, and timing of treatment. Data were entered using Epi-Info 6.04 (Centers for Disease Control and Prevention, Atlanta, GA, USA) and analysed in Stata (V.8 College station, TX, USA). Descriptive statistics are presented and selected characteristics were assessed using one way analysis of variance.

Ethical approval was granted by the University of the Witwatersrand, Johannesburg, human subject ethics committee.

\section{RESULTS}

In total, 18128 records were reviewed and, of these, 188 women were found to be syphilis positive. This showed a prevalence rate of $1.03 \%$. The median gestation of women at their first visit was 26 weeks with a range of 12-40 weeks, and $17 \%$ presented for their first antenatal visit at 30 weeks or later. Few women attended before 20 weeks gestation $(10.7 \%)$.

Almost two thirds $(64.8 \%)$ of women received all three doses, $5.8 \%$ received two doses, $13.2 \%$ received one dose, and $15.9 \%$ of women received no treatment (table 1).

Number of doses of treatment was significantly associated with age of gestation at first ANC visit $(p=0.029)$ with women presenting later in their pregnancy being less likely to receive all three doses (table 2 ).

The median time elapsed from testing to receiving the first dose of treatment was 34 days (range 1-169 days). The majority $(81 \%)$ were first treated after 14 days and almost a fifth $(18 \%)$ waited for at least 2 months from testing to commencement of treatment. The median time between first and second doses was 7 days and between second and third doses was 7 days. In $6 \%$ of cases the dose was given earlier than 7 days between the first and second dose and second and third dose. Almost half of women (49\%) received the second dose later then 7 days and $28 \%$ received the third dose later than 7 days. There was no significant association between clinic and completion of treatment in the 30 clinics that referred to the hospital.

Just over half $(n=110)$ of maternity records gave information on titre (table 3 ). Over a third $(39.4 \%)$ of titres were 1 in 2 dilution and over a fifth $(22.9 \%)$ were $1: 32$.

\section{DISCUSSION}

The prevalence of syphilis was low in this record review $(1.03 \%)$. This is consistent with surveillance data from the province in the same year $(1.3 \%) .^{5}$ The national prevalence has decreased from previous years and the prevalence in 2001 was $2.8 \%$ declining from $4.9 \%$ in 2000 . The blood for the syphilis test is taken at the first ANC visit; however, women in this population tend to present late in pregnancy. Only $10 \%$ of women start ANC before 20 weeks gestation, which
Table 2 Number of doses of treatment by age of gestation at first visit

\begin{tabular}{lll}
\hline \multirow{2}{*}{ No of doses } & \multicolumn{2}{l}{ Age of gestation at first visit (weeks) } \\
\cline { 2 - 3 } & Mean & $\mathbf{n}=169^{*}$ \\
\hline 0 & 28 & 20 \\
1 & 27 & 23 \\
2 & 24 & 10 \\
3 & 25 & 116 \\
\hline \multirow{2}{*}{ * 9 records missing for age of gestation. }
\end{tabular}

\begin{tabular}{lcl} 
Table 3 & Titres of positive cases & \\
\hline RPR titres & $\%$ & $\mathbf{n}=110$ \\
\hline $1: 2$ & 39.4 & 43 \\
$1: 4$ & 24.7 & 27 \\
$1: 8$ & 9.1 & 10 \\
$1: 16$ & 4.5 & 5 \\
$1: 32$ & 22.9 & 25 \\
\hline
\end{tabular}

limits time to complete treatment should she be found to be positive.

Almost two thirds of women completed their treatment, receiving all three doses. This is higher then reported in previous South African studies ${ }^{11} 12$ and may indicate an improvement; however, it could be that the study site was in an urban area with better infrastructure than the previous reported studies which were conducted in mainly rural settings. The number of treatment doses was significantly associated with age of gestation at first visit. Women who received no treatment presented on average 3-4 weeks later for their first visit than the women receiving all three doses. Data also showed that median time to the first dose after testing was 34 days. The national ANC guidelines state a woman should return to the clinic 2 weeks after testing to receive her results; however, women often only get results at their next routine ANC visit. If results are not routinely returned to clinics from laboratories within 2 weeks, providers may be reluctant to ask women to return at this time.

When the two further doses are added an additional 2 weeks are required to complete treatment. This indicates that a woman presenting at 28 weeks may be unlikely to complete her treatment as time taken to complete treatment can often be up to 3 months. One South African study in the same area found that $15 \%$ of women attended so late in pregnancy that it would not have been possible to complete treatment before delivery. ${ }^{13}$ In a third of cases the first dose was not given until 2 months after testing. Once the first dose was given it appeared that the next two doses were mainly given at the correct intervals with median time being 7 days in each case. There were, however, women who had the dose too early and almost half were delayed for the second dose and a third were delayed for the third dose.

A significant proportion of women are high titre positives; these have a strong association with adverse pregnancy outcomes, and thus improving compliance may have a significant impact on reducing perinatal deaths, stillbirths, and preterm births. ${ }^{14} 15$

In some cases, information was missing such as gestation age at first visit and titre indicating that recording of routine data needed improvement.

There are no current plans in South Africa to consider the WHO recommended one dose treatment for all stages of syphilis except late latent syphilis. ${ }^{16}$ Antenatal cards currently do not have pregnancy outcome reported on them and it 


\section{Key messages}

- Late presentation for first antenatal visit may impact on the possibility of completing the three dose treatment with penicillin for syphilis

- High rates of testing for syphilis do not always indicate a successful programme. Treatment completion should be used in addition to testing to monitor programme performance

- Recording data is key to monitor performance. Antenatal cards should record information on birth outcomes in cases where women were not tested. In addition, improvement is needed in recording of routine data, such as age of gestation and titre

would thus be difficult to ascertain outcomes for women receiving three doses and those who did not. However, these results would still have been confounded by a number of factors difficult to control for such as gestation when syphilis was acquired by the mother, gestational age at first visit, and presence of other reproductive tract infections. A cost effectiveness analysis of using a one dose versus three dose approach to treatment should be conducted to inform the policy on screening and treatment. This analysis should include presumptive treatment of all antenatal women as an option. In the meantime, there is a need to encourage early starts to ANC and prompt commencement of treatment. Implementation of on-site or rapid testing at antenatal care facilities my reduce time from testing to starting treatment and ensure that more women complete treatment before delivery.

Combining both negative and positive results the records indicated that almost all women $(>90 \%)$ were tested for syphilis. In this respect the programme is performing well. The success of this programme is therefore compromised by the poor treatment compliance.

Efforts are needed to improve compliance with the treatment and encourage women to come early for antenatal care. Healthcare workers in labour and maternity wards should be aware that some of the women coming to deliver may not have completed their treatment and may require treatment of the infant. The training curriculum for healthcare workers also needs to be strengthened to include accurate documentation of treatment and partner involvement.

Clinics should report levels of compliance to health informatics for monitoring purposes.

Traditionally, antenatal care services have excluded men even though they often influence women's access to health services. There are inconsistencies in treatment of the partner where a woman is syphilis positive with some men given three doses while others receive only one dose. ${ }^{13}$ Male involvement in antenatal and postnatal care has been found to be feasible, acceptable, and effective in South Africa in a pilot setting. However, this is not routinely done (Men in maternity: improving access to sexual and reproductive health services by involving men in their partners antenatal and postnatal care, submitted for publication).

Given the current context of HIV in South Africa, the recent widespread roll out of prevention of mother to child transmission (PMTCT) programmes and the need to screen for both syphilis and HIV in pregnant women, there may be possible links between these two programmes that could be strengthened. ${ }^{17}$ In addition, the newer PMTCT programmes could learn from the successes and challenges of the longer standing syphilis screening programme. ${ }^{13}$

\section{Limitations}

Data collected were limited to what was available on the maternity record cards and in some cases some data were missing from the cards. In addition, partner notification and treatment are not recorded on the antenatal cards and it is thus difficult to establish the proportion of partners treated.

\section{ACKNOWLEDGEMENTS}

The authors thank USAID for funding the study. We would also like to acknowledge the contribution of Ms Dolly Ngwenya of the Reproductive Health Research Unit for her work in collecting the data for this study and Dr Ian Askew of the Population Council (Frontiers) for his comments on the draft version.

\section{CONTRIBUTORS}

SMu planned and managed the study, analysed the data, and was primary writer of this article; MB was involved in study supervision, data management and analysis and was involved in writing the paper with SMu; SMs was involved in data collection and analysis and contributed to writing of the paper.

\section{Authors' affiliations}

S Mullick, Population Council, Frontiers in Reproductive Health, Hyde Park Lane Manor, EG001 Edinburgh Gate, Hyde Park, Box 41 1744, Craighall 2024, Johannesburg, South Africa

M Beksinksa, S Msomi, Reproductive Health and HIV Research Unit, Department of Obstetrics and Gynaecology, University of the Witwatersrand, South Africa, Suite 1301, Maritime House, 143 Salmon Grove, Durban, South Africa

\section{REFERENCES}

1 Fleming DT, Wasserheit. From epidemiological synergy to public health policy and practice: the contribution of other sexually transmitted diseases to sexual transmission of HIV infection. Sex Transm Infect 1999;75:3-17.

2 Temmerman M, Gichangi P, Fonck K, et al. Effect of a syphilis control programme on pregnancy outcome in Nairobi, Kenya. Sex Transm Infect 2000;76:117-21.

3 Azeze B, Fantahun M, Kidan K, et al. Seroprevalence of syphilis among women attending antenatal clinics in rural hospital in North West Ethopia. Genitourin Med 1995;71:347-50.

4 Bam RH, Cronje HS, Muir A, et al. Syphilis in pregnant patients and their offspring. Int J Gynecol Obstet 1994;44:113-18.

5 Summary report: National HIV and syphilis antenatal sero-prevalence survey in South Africa 2002

6 Hira SK, Bhat GJ, Chikamata DM. Syphilis intervention in pregnancy: Zambian demonstration project. Genitourin Med 1990;66:159-64.

7 Stray-Perderson B. Economic evaluation of maternal screening to prevent congenital syphilis. Sex Transm Dis 1983;10:167-72.

8 Delport SD, Rothberg AD. Congenital syphilis-now a notifiable disease. S Afr Med J 1992;81:288-9.

9 Ingraham NR. The value of penicillin alone in the prevention and treatment of congenital syphilis. Acta Dermatol Venereol 1951;31(Suppl 24):60-88.

10 National Department of Health. Guidelines for maternity care in South Africa. Pretoria, South Africa: National Department of Health, 2002.

11 Wilkinson D, Sach M, Connolly C. Epidemiology of syphilis in pregnancy in rural South Africa: opportunities for control. Trop Med Int Health 1997;2:57-62.

12 Rotchford K, Lombard K, Zuma K, et al. Impact on perinatal mortality of missed opportunities to treat maternal syphilis in rural South Africa: baseline results from a clinic randomized controlled trial. Trop Med Int Health 2000;5:800-4.

13 Beksinska ME, Mullick S, Kunene B, et al. A case study of antenatal syphilis screening in South Africa: successes and challenges. Sex Transm Dis 2002;29:32-7.

14 McDermott J, Steketee R, Larsen S, et al. Syphilis-associated perinatal and infant mortality in rural Malawi. Bull World Health Organ 1993;71:773-80.

15 Watson-Jones D, Gumodoka B, Weiss H, et al. Syphilis in pregnancy in Tanzania II. The effectiveness of antenatal syphilis screening and single-dose benzathine penicillin treatment for the prevention of adverse pregnancy outcomes. J Infect Dis 2002;186:948-57.

16 World Health Organization. Guidelines for the management of sexually transmitted infections, WHO/HIV_AIDS/2001.01. Geneva: WHO, 2001.

17 Mullick S, Broutet N, Htun Y, et al. Controlling congenital syphilis in the era of HIV/AIDS. Commentary. Bull World Health Organ 2004;82:431-2. 